\title{
An Adaptive Impedance Matching System for Mobile Communication Antennas
}

\author{
Ichirou IDA* Jun-ichi TAKADA Takeshi TODA Yasuyuki OISHI \\ Tokyo Institute of Technology \\ 2-12-1, O-okayama, Meguro \\ Fujitsu Limited \\ YRP R\&D Center, 5-5, Hikari-no-Oka
Yokosuka, Kanagawa 239-0847, JAPAN \\ ida@ap.ide.titech.ac.jp, takada@ide.titech.ac.jp, ttoda@jp.fujitsu.com, yasu@labs.fujitsu.com
}

\begin{abstract}
An adaptive impedance matching system is proposed. This system employs only a simple algorithm for convergence, therefore does not need any complicated mathematical formulation for modeling of the system itself as well as its nonlinear control elements. Also the proposed system utilizes only simple digital circuits for timing generation and basic analog circuits. Even with a small range of capacitance for control element in a matching circuit, the improvement in the mismatch is observed by $2-3 \mathrm{~dB}$ over an impedance matching circuit with fixed capacitances.
\end{abstract}

\section{Introduction}

In recent years, mobile communication systems and terminals have been rapidly developed and demands for the higher data rate are incessant. By nature, however, the mobile terminals are subject to unexpected environmental changes e.g., a human body and other objects in the vicinity of the users. The problems here are that those objects near the mobile antennas cause the change in the antenna impedance, because the current distribution on the antenna is affected by them [1]. Thus, there exists a mismatch between the antenna impedance and the input impedance of the RF front end. In the case of transmission, such impedance mismatch leads to a damage of the power amplifier due to the power reflected back to its input, and it also causes a shorter battery lifetime due to excessive energy consumption. While in the receiving path, it causes degradation in the carrier to noise ratio (CNR), although employment of a higher data rate should be supported by a higher CNR. Thus, such impedance mismatch should be avoided as much as possible.

One way to avoid the mismatch is to give multiple antennas to a mobile terminal and to selectively use the antenna, which is least affected by the nearby objects. This method is, however, not easy to implement, while the adaptive adjustment of the circuit constants in the matching circuit seems to be much simpler.

In addition, there has been a method, which utilizes an L, C bank to realize a good impedance matching with combination of those inductors and capacitors. In this system, however, only a limited resolution of the impedance matching is available. Besides the use of varactors combined with driving motors has a prohibitive volume for most of mobile communication terminals.

Also an adaptive impedance matching systems with the steepest gradient algorithm for convergence has been reported [2]. When some mathematical formulation is required, however, a precise modeling of the overall system and control elements are vital. This is often difficult, therefore sometimes causes the deviation of the theory from the reality.

Considering the problems above mentioned, we propose an adaptive impedance matching system, which utilizes only simple digital circuit for generation of the timing signals and basic analog circuits. The adaptive control is conducted in a sequential manner, and it does not need any complicated mathematical formulation.

In this paper, we first show the configuration of the system as well as 
some merits of the proposed system. Second, some simulational results are depicted before the conclusions.

\section{Configuration of the system}

Figure 1 shows the configuration of the system. The system is now intended to be used in the transmitting path. It consists of (1) Matching circuit, (2) Mismatch measurement circuit (directional coupler), (3) Switch, (4) Timing generator for switching, and (5) Time constant generator (RC low-pass filter).

The varactors (variable capacitors) are used for the control elements in the matching circuit. In this configuration, however, the available information to be used for the impedance matching is only the absolute value of the reflection coefficient between the RF source and



Fig. 1 Configuration of the proposed adaptive matching system

the input of the matching circuit. Thus as long as we cannot measure the phase information of the reflection coefficient, we don't know the correct direction of the control to minimize the mismatch. To solve this problem, we employ a test signal to observe if applying the test signal increases or decreases the mismatch. Then we know for the first time if the direction of the control was correct or wrong.

The adaptive matching protocol of the proposed system is as follows: (a) The latest value of the mismatch is measured through the detection circuit (2). (b) The switch (3) is turned on, i.e. the control voltage to the varactor 1
(VC1) is increased. (c) The mismatch is again measured right after turning on the switch. Here, if the mismatch has been increased compared with the previous measurement, then the system recognizes that the direction of the control was wrong, and the switch is turned off to decrease the control voltage to the $\mathrm{VC} 1$. If the mismatch has been decreased compared with the previous measurement, the system recognizes that the direction of the control was correct, and the switch is kept turned on until the end of the control frame for the VC1. During the VC1 frame, the control voltage to the $2^{\text {nd }}$ varactor $\mathrm{VC} 2$ is held to the value at the end of the last frame of the $\mathrm{VC} 2$. This voltage holding is conducted by the sample and hold circuits. After the VC1 frame is finished, the control voltage for the $\mathrm{VC} 1$ is held and the VC2 frame is commenced.

The advantages of the proposed system are as follows:

(1) Compared with systems, which employ the steepest decent algorithm for optimization, the proposed system does not require such a complicated mathematical modeling.

(2) When a mathematical modeling is necessary, the control elements of the matching circuit should also be modeled precisely. In this case, nonlinearity of the varactors and etc. can be very problematic for a good convergence, because formulation of such nonlinear elements is difficult. The proposed system can accept also such nonlinear control elements.

(3) In addition, varactors with any range of capacitance are applicable to the proposed system, because its criterion for the control is to reduce the mismatch as much as possible, thus no goal for convergence like in the steepest decent method. In other words, it simply tries to match the impedance with the available capacitance range of the varactors. 


\section{Simulation results}

Some simulational consideration was made by using Simulink from The MathWorks Inc. Figure 2 shows the simulation result for the improvement of the mismatch with the adaptive impedance matching system. Here it is evaluated by observing the reflection coefficient between the matching circuit connected with an antenna and the RF front end with 50ohms of characteristic impedance. In Fig. 1, the reflected power due to the mismatch can be increased in the following two cases: (1) Worse mismatch, and (2) Increase in the transmitted power. Use of the directional coupler as the mismatch measurement circuit in Fig. 1, however, cannot discriminate between (1) and (2) above. Therefore we need to tap the two outputs, i.e. the isolation and coupling, from the directional coupler, and to divide the isolation by the coupling. Then, we can measure only the increase in the mismatch, not in the transmitted power.

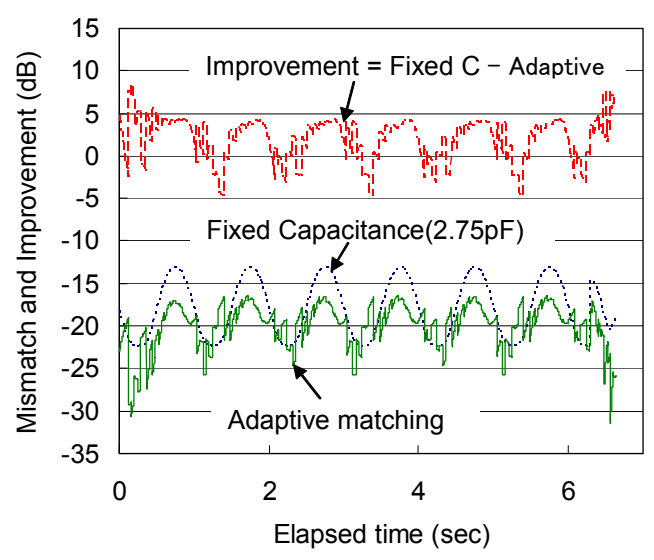

Fig. 2 Mismatch and its improvement (only antenna resistance is varied)

The impedance matching system without variable elements is also simulated for comparison. The parameters for the simulation are: Operating frequency $2.45 \mathrm{GHz}$, Range of capacitance for varactors $2.5 \mathrm{pF}$ $3.0 \mathrm{pF}$, Fixed capacitance for the compared system $2.75 \mathrm{pF}$. In Fig. 2, time characteristics of the mismatch by the adaptive system and fixed capacitance system are compared. Also the improvement in the mismatch value is illustrated as the subtraction of the value of the adaptive system from that of the fixed capacitance system. Here, the input resistance of the antenna input impedance $Z_{\mathrm{a}}$ is sinusoidally fluctuated with a range of $50 \pm 10 \mathrm{ohms}$. The frequency of the fluctuation is $1 \mathrm{~Hz}$. The imaginary part of the $Z_{a}$ is fixed to $40 \mathrm{ohms}$. The inductance $L$ in the matching circuit is $2.9 \mathrm{nH}$. The time constant for the time constant generator is $0.1 \mathrm{sec}$. The range of the fluctuation for $Z_{\mathrm{a}}$ is assumed to move only within the upper half of the Smith chart, i.e. the antenna impedance $Z_{\mathrm{a}}$ is always inductive. We confirmed it with an experiment, where a monopole antenna (rooftop type for automotive covered with resin) was placed on a metallic table, on a concrete floor, and in the vicinity of a human body. The monopole antenna was even grasped and measured. Thus we use the above assumption of the inductive antenna input impedance $Z_{\mathrm{a}}$ in the present consideration.

Figure 3 shows the time integration of the improvement in Fig. 2. The amount of the integration is decreased while the mismatch of the adaptive system exceeds that of the fixed system. Through dividing the integrated value

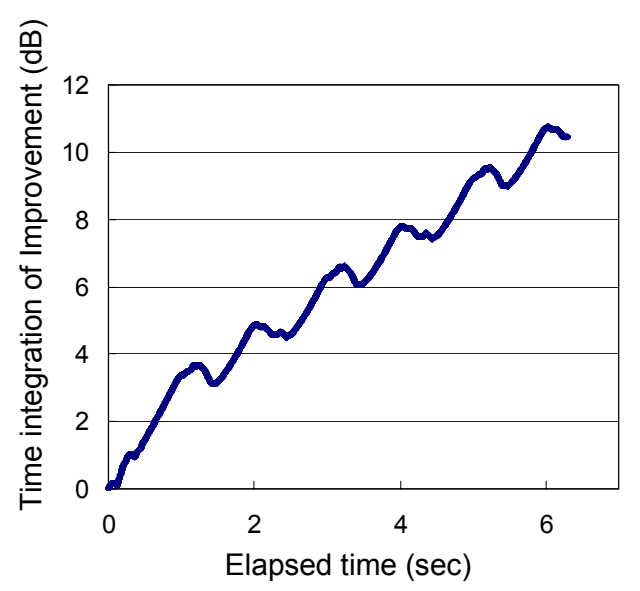

Fig. 3 Time integration of improvement in Fig. 2 
by the elapsed time, we obtain the average improvement of the mismatch.

In Fig. 3, about $1.8 \mathrm{~dB}$ of the mismatch improvement is achieved at 6 sec.

Figure 4 shows another result for the mismatch improvement. This time, only the imaginary part of the antenna input impedance $Z_{\mathrm{a}}$ is sinusoidally fluctuated with a range of $30 \pm 10 \mathrm{ohms}$. The frequency of the fluctuation is $1 \mathrm{~Hz}$. The real part of the $Z_{\mathrm{a}}$ is fixed to $50 \mathrm{ohms}$. The inductance $L$ is $2.8 \mathrm{nH}$. The time constant for the time constant generator is $0.13 \mathrm{sec}$.

Figure 5 shows the time integration of the mismatch improvement in Fig. 4. The average mismatch improvement is calculated to be about $2.7 \mathrm{~dB}$ at 6 sec.

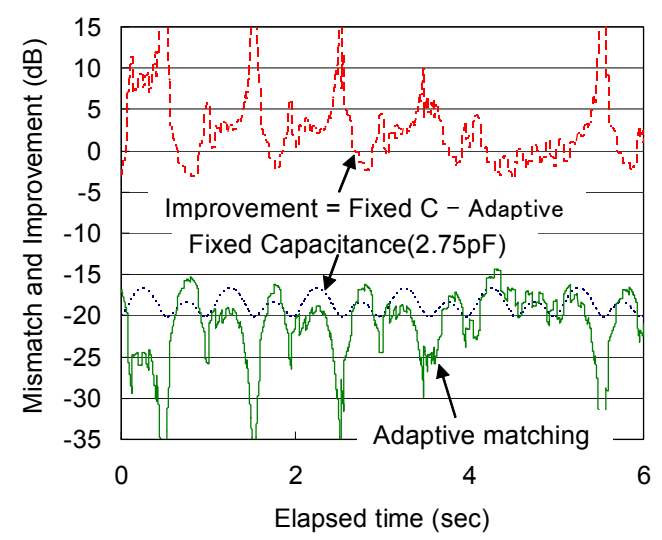

Fig. 4 Mismatch and its improvement (only antenna reactance is varied)

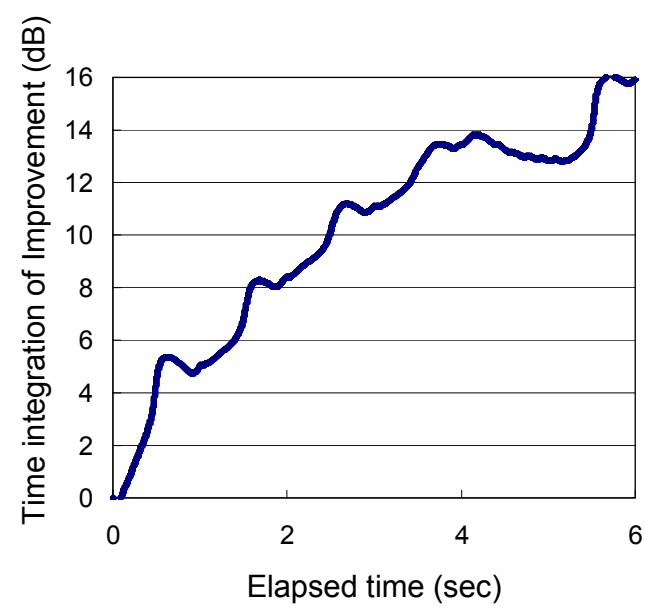

Fig. 5 Time integration of improvement in Fig. 4
In general, we confirmed that the smaller the amplitude of the fluctuation of the antenna impedance is, the better the adaptive matching works.

\section{Conclusions}

An adaptive impedance matching system for mobile communication has been proposed. The improvement of the mismatch by $2-3 \mathrm{~dB}$ over the fixed matching circuit has been confirmed with the simulation. The relationship between the range of capacitance for varactors and the improvement in the mismatch should be considered as a future study.

\section{Acknowledgement}

This research was supported by the Telecommunications Advancement Organization of Japan.

\section{$\underline{\text { References }}$}

[1] K. Ogawa, T. Matsuyoshi, and K. Monma, "An analysis of the performance of a handset diversity antenna influenced by head, hand, and shoulder effects at $900 \mathrm{MHz}$ : Part I - effective gain characteristics," IEEE Trans. Vehicular Technology, vol. 50, no. 3, pp. 830-844, May 2001.

[2] T. Takahashi, Y. Koyanagi, K. Ogawa, and K. Ito, "Automatic impedance matching of an active antenna near the human operator by the steepest gradient algorithm," Proceedings of the 2003 IEICE general conference, p. 61, B-1-61, March 2003 (in Japanese). 